\title{
Quasiparticles in the Pseudogap Phase of Underdoped Cuprate
}

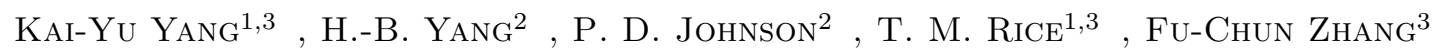 \\ ' 1 Institut für Theoretische Physik, ETH Zürich,CH-8093 Zürich, Switzerland \\ 2 Condensed Matter Physics and Materials Science Department, Brookhaven National Laboratory, Upton, NY 11973, \\ $U S A$ \\ 3 Center for Theoretical and Computational Physics and Department of Physics, The University of Hong Kong, Hong \\ Kong SAR, China
}

PACS 74.72.-h - Cuprate superconductors

PACS 74.20.Mn - Nonconventional mechanisms

PACS 79.60.-i - Photoemission and photoelectron spectra

\begin{abstract}
Recent angle resolved photoemission [1] and scanning tunneling microscopy [2] measurements on underdoped cuprates have yielded new spectroscopic information on quasiparticles in the pseudogap phase. New features of the normal state such as particle-hole asymmetry, maxima in the energy dispersion and accompanying drops in the spectral weight of quasiparticles agree with the ansatz of Yang et al. for the single particle propagator in the pseudogap phase. The coherent quasiparticle dispersion and reduced asymmetry in the tunneling density of states in the superconducting state can also be described by this propagator.
\end{abstract}

The anomalous electronic properties of the pseudogap phase in underdoped cuprates continue to challenge theory. Angle resolved photoemission (ARPES) measurements of the Fermi surface in the pseudogap state show only disconnected Fermi arcs centered on the nodal directions. [3-5] A recent high resolution ARPES study on underdoped BSCCO by Yang and collaborators [1] examined the spectra below and above the chemical potential $\mu$ by carefully dividing out the Fermi function. They found that the spectra displayed particle-hole asymmetry away - from the nodal direction in the pseudogap state but not in optimal doped samples, so that the asymmetry is a property of the pseudogap phase. Scanning tunneling mi' croscopy (STM) by Kohsaka and collaborators [2] has determined the coherent quasiparticle dispersion in a wide range of hole densities in the superconducting state, by an ingenious analysis of the voltage dependent spatial interference patterns. [6] The asymmetry in the tunneling density of states measured by STM is weaker due to the inherent particle-hole symmetry of superconductivity. Recent angle integrated photoemission (AIPES) experiments on underdoped samples [7] found the density of states varied linearly with energy in contrast to the constant value that appears at a band edge in two dimensions. In this letter we analyze ARPES, STM and AIPES results using an ansatz for the single particle propagator proposed earlier by three of us. Key properties of the quasiparticles (QP) in this propagator, such as maxima in the energy dispersion in the normal pseudogap state, the connection between the QP dispersion in the superconducting state and the hole density, are directly confirmed by these experiments. An alternative explanation of particle-hole asymmetry in ARPES experiments has been proposed by Anderson [8].

As the hole density, $x$, crosses the underdoped pseudogap region of the phase diagram, the cuprates crossover from a full Fermi surface metal at overdoping to a Mott insulator at stoichiometry. A Mott insulating state is driven by strong onsite Coulomb repulsion and not per se by translational symmetry breaking. The stoichiometric underdoped cuprate $\mathrm{YBa}_{2} \mathrm{Cu}_{4} \mathrm{O}_{8}$, shows no sign of a charge or spin density modulation on either $\mathrm{Cu}$ or $\mathrm{O}$ sites. [9] It is therefore desirable to examine theories where the truncated Fermi surface appears as a precursor to the Mott state without translational symmetry breaking. An example is the 2D array of 2-leg Hubbard ladders studied by Konik, Rice and Tsvelik (KRT). [10] They showed that lines of zeros in the propagator $G^{R}(\boldsymbol{k}, \omega=0, x)$ associated with the charge gap at half-filling, enclosing a commensurate area of $1 \mathrm{el} /$ site, do not move with light doping. The Luttinger Sum Rule on the area enclosed by the condition $\operatorname{Re}\left[G^{R}(\boldsymbol{k}, \omega=0, x)\right]>0$ is fulfilled with the Fermi surface truncated to small hole pockets. 
Table 1: The bare hopping parameters $t_{0}^{\prime}, t_{0}^{\prime \prime}$ and the superexchange $J$ are quoted in units of the nn value $t_{0}=0.3 \mathrm{eV}$. The hole density determines the pocket area and is consistent with the value determined independently [1].

\begin{tabular}{|c|c|c|c|c|c|c|}
\hline \hline$t_{0}^{\prime}$ & $t_{0}^{\prime \prime}$ & $J$ & $x$ & $\Delta^{0}$ & $\Delta^{0}(x)$ & $\chi$ \\
-0.3 & 0.2 & $1 / 3$ & 0.12 & 0.5 & $\Delta^{0}\left(1-\frac{x}{0.2}\right)$ & 0.338 \\
\hline \hline
\end{tabular}

A relevant question is whether similar behavior can occur in a fully 2D system. Honerkamp and coworkers [11] argued for such behavior from the similarities in the flow of the response functions in functional renormalization group analyses of Hubbard models near half-filling. Yang, Rice and Zhang [12] (YRZ) proposed an adaptation of the KRT approach to the lightly doped $t$ - $J$ model. They introduced a self-energy, $\Sigma^{R}(\boldsymbol{k}, \omega, x)$, which diverges at $\omega=0$ on a surface spanned by elastic particle-particle umklapp scattering analogous to the behavior of the ladder model. In the 2D square lattice this umklapp surface is a diamond connecting antinodal points on the Brillouin zone boundary. Note this umklapp surface appears as the energy gap surface also in the case of wider Hubbard ladders with more than 2 legs. [13]

YRZ took over the form for the gap function $\Delta^{R}(\boldsymbol{k}, x)$ from the renormalized mean field theory of Zhang et al. [14] for the resonant valence bond (RVB) state $[15,16]$ of the strong coupling $2 \mathrm{D} t-J$ model and proposed an ansatz for the single particle propagator:

$$
G^{R}(\boldsymbol{k}, \omega, x)=g^{t}(x) /\left[\omega-\xi_{\boldsymbol{k}}-\Sigma^{R}(\boldsymbol{k}, \omega, x)\right]+G_{i n c}
$$

where $\Sigma^{R}(\boldsymbol{k}, \omega, x)=\left|\Delta^{R}(\boldsymbol{k}, x)\right|^{2} /\left(\omega+\xi_{\boldsymbol{k}}^{(0)}\right)$ is the RVB self-energy. The energy $\xi_{\boldsymbol{k}}^{(0)}=-2 t(x)\left(\cos k_{x}+\cos k_{y}\right)$ vanishes on the umklapp surface. The renormalized dispersion $\xi_{\boldsymbol{k}}=\xi_{\boldsymbol{k}}^{0}-4 t^{\prime}(x) \cos k_{x} \cos k_{y}-2 t^{\prime \prime}(x)\left(\cos 2 k_{x}+\right.$ $\left.\cos 2 k_{y}\right)-\mu$ includes hoppings out to $3^{\text {rd }}$ nearest neighbor with hole density dependent coefficients $t(x)=g^{t}(x) t_{0}+$ $3 g^{s}(x) J \chi / 8, t^{\prime}(x)=g^{t}(x) t_{0}^{\prime}$, and $t^{\prime \prime}(x)=g^{t}(x) t_{0}^{\prime \prime}$. The Gutzwiller factors $g^{t}(x)=2 x /(1+x)$ and $g^{s}(x)=$ $4 /(1+x)^{2}$ account for the no-double occupation projection on the kinetic and superexchange terms in the $t-J$ model. The RVB energy gap takes the form $\Delta^{R}(\boldsymbol{k}, x)=$ $\Delta^{(0)}(x)\left(\cos k_{x}-\cos k_{y}\right) \cdot \chi$ corresponds to the weakly doping dependent homogeneous amplitude $\left\langle c_{i, \sigma}^{\dagger} c_{j, \sigma}\right\rangle$. Parameters are quoted in Table 1 .

As discussed by YRZ the Fermi surface in the pseudogap regime with divergent $G^{R}(\boldsymbol{k}, \omega=0, x)$ has 4 pockets centered on the nodal directions enclosing a total area related to the hole density. [12] ARPES experiments [1,3-5] show arcs rather than closed pockets, but as YRZ pointed out the QP weight in $G^{R}$ varies rapidly with very small values on the back sides of the pockets, which can account for the failure to observe these parts of the pockets. Many experimental features, e.g. the slow variation of the nodal Fermi velocity $v_{F}$ and Drude weight scaling with hole density, are reproduced by the YRZ propagator. [12] The umklapp sur-
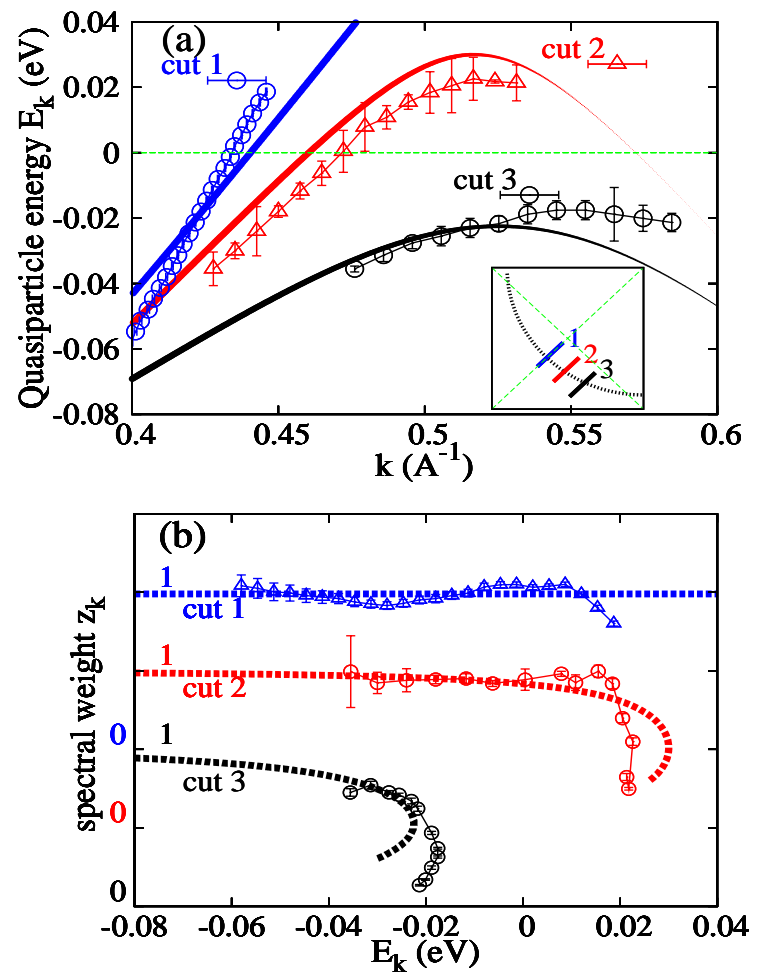

Fig. 1: (Color online) Comparisons between (a) QP dispersion $E_{\boldsymbol{k}}$, (b) spectral weight $z_{k}$, from the YRZ propagator and the values obtained from the ARPES results by Yang et al. [1] Error bars reflect the uncertainties in the fitting procedures; $z_{\boldsymbol{k}}$ at low energies in cuts 2,3 have large error bars due to uncertainties in the choice of the rising background.

face along which the energy gap opens up, lies above $\mu$, causing particle-hole asymmetry in the QP spectra in the pseudogap state. The spectra are symmetric only along the nodal directions where $\Delta^{R}(\boldsymbol{k}, x)=0$, and the asymmetry increases away from the nodal directions.

The spectra observed by Yang et al [1] along three cuts (see inset in Fig,1) in the pseudogap phase $(T=140 \mathrm{~K})$ are shown in the Brillouin zone in Fig.2(a). They are fits to a broadened Lorentzian plus a background term to be defined below. The fitting uses an implementation of the nonlinear least-square Marquardt-Levenberg algorithm. We determine the QP dispersion, $E_{\boldsymbol{k}}$, weight $z_{\boldsymbol{k}}$, and inverse lifetime $\gamma_{\boldsymbol{k}}$ for each $\boldsymbol{k}$-value from the form.

$$
A(\boldsymbol{k}, \omega)=\operatorname{Im}\left[z_{\boldsymbol{k}} /\left(\omega-E_{\boldsymbol{k}}+i \gamma_{\boldsymbol{k}}\right)\right]+A^{B}(\boldsymbol{k}, \omega)
$$

For the background, momentum distribution curves (MDC) of cut 1 are well fitted with an almost constant background $A^{B}(\boldsymbol{k}, \omega)$, for energy distribution curves (EDC) of cuts 2 and 3 we use a broadened step function which rises at lower energies well below $\mu$,

$$
A^{B}(\boldsymbol{k}, \omega)=y_{\boldsymbol{k}}\left(\tanh \left[\lambda_{\boldsymbol{k}}\left(\Omega_{\boldsymbol{k}}-\omega\right)\right]+1\right)+\alpha_{\boldsymbol{k}}
$$

The fitted MDC(EDCs) for the three cuts are shown in the upper panels of Fig $2(\mathrm{a})$, and the two components, 


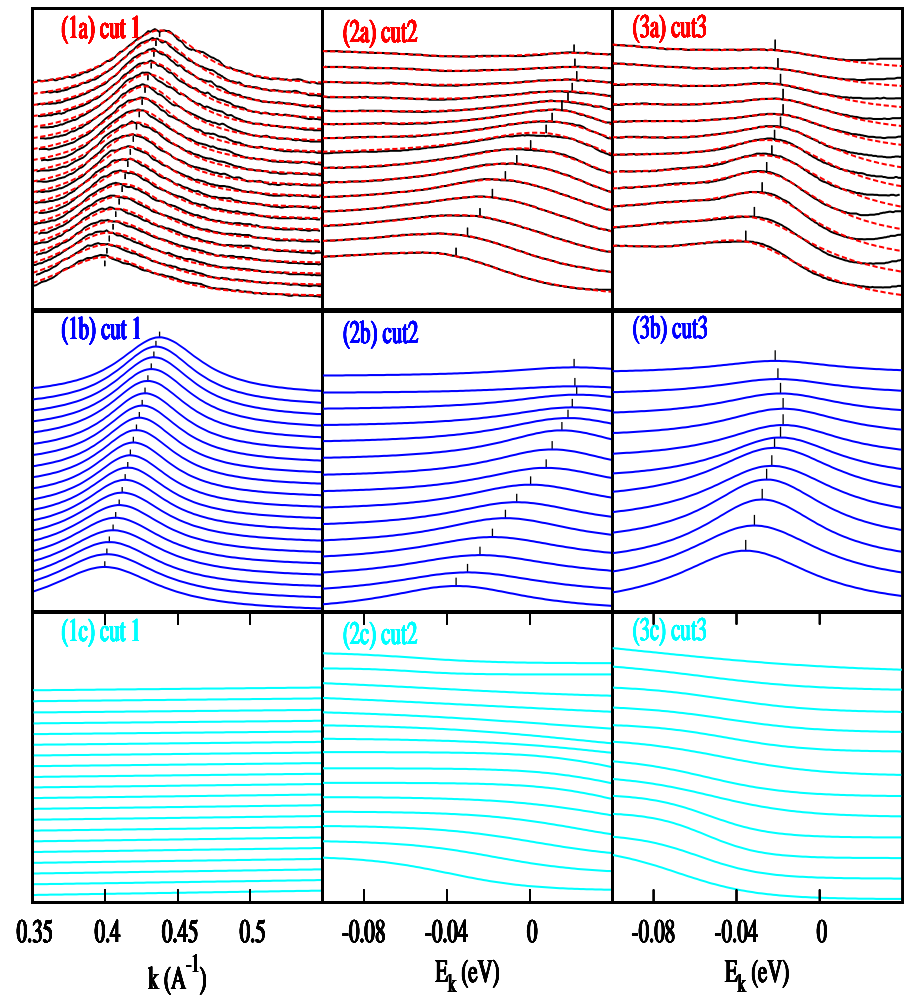

Fig. 2: (Color online) Fits to the ARPES spectra along the cuts (1-3) (see inset of Fig 10 [1]. In (1a) the MDC of cuts 1 and in $(2 / 3 a)$ the EDC of cuts 2 and 3 are shown, the experimental data are the solid black lines, and the fitted $A(\boldsymbol{k}, \omega)$ are the dashed red lines. Individual components of the fits are displayed in (b) for QP Lorentzian peaks and (c) for smooth background $A^{B}(\boldsymbol{k}, \omega)$.

the Lorentzian peak and background, in the lower panels (b/c). For cut 1 along the nodal directions we note that the fitting to a broadened Lorentzian with an almost constant background works well for all energies. For cuts 2-3 EDC fitting, as $\boldsymbol{k}$ moves away from $\boldsymbol{k}_{F}$, the background component $A^{B}(\boldsymbol{k}, \omega)$ increases at lower energies. We believe this increase arises from contamination from adjacent $\boldsymbol{k}$-values, due to the enhanced broadening at low energies. Since our interest focusses on energies near $\mu$ we believe this choice of background which is constant near $m u$ is reasonable.

Fig 1 shows the results for the QP properties of interest. The QP dispersion, $E_{\boldsymbol{k}}$ in Fig:1(a) rises linearly with $\boldsymbol{k}$ along the nodal cut 1 . Particle-hole asymmetry is evident in cuts 2 (3) with a maxima in $E_{\boldsymbol{k}}$ lying above (below) $\mu$, respectively. Note that these maxima are not at the boundary of the reduced Brillouin zone as the case would be in the presence of a broken translation symmetry, a point emphasized by Yang et al. [1] The QP weight, $z_{\boldsymbol{k}}$, represented in the dispersion plot as the line width, is plotted in Fig प(b). We see an almost constant $z_{\boldsymbol{k}}$ for cut 1 along the nodal direction, but a fall off at the maximum in $E_{\boldsymbol{k}}$ for cuts 2 and 3 in agreement with the YRZ ansatz.

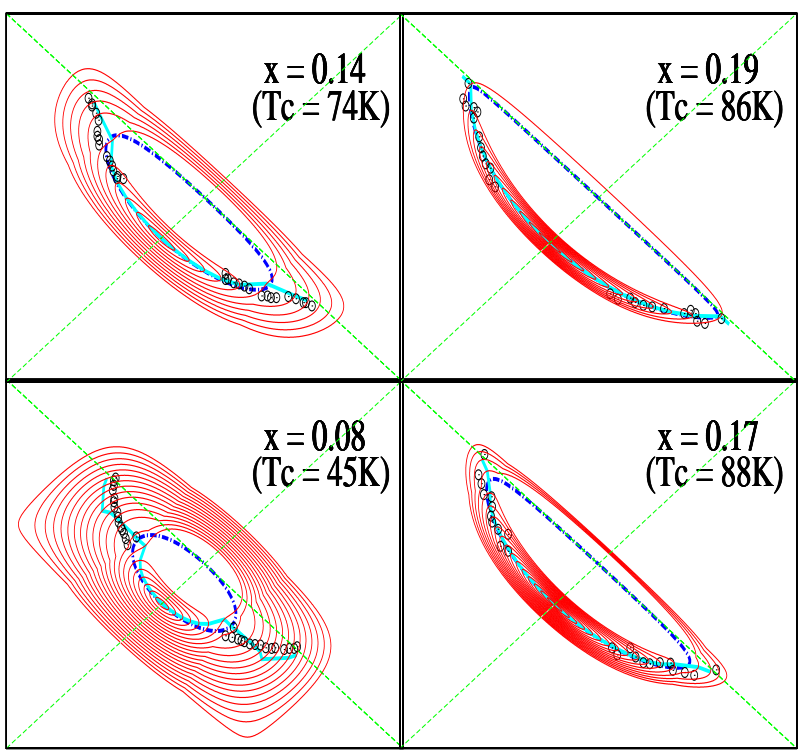

Fig. 3: (Color online) The red curves are the contours of the constant QP energy below and close to the Fermi energy. The black dots are the turning points deduced from STM interference data. The dashed blue lines are the hole Fermi pocket in the normal pseudogap state. The cyan curves are the turning points of the iso-energy contour of the corresponding band in the SC state. Note only the turning point of the iso-energy contour with the largest spectral weight are shown.

The inverse lifetime $\gamma_{\boldsymbol{k}}$ is essentially constant indicating substantial inelastic scattering at this elevated temperature of $140 \mathrm{~K}$. To summarize with reasonable parameters the phenomenological YRZ propagator gives a good fit to the asymmetry of ARPES spectra and describes both the maxima in the QP dispersion, $E_{\boldsymbol{k}}$, away from the nodal direction and the accompanying drop off in the weight, $z_{\boldsymbol{k}}$.

In their original discussion of the ARPES spectra, Yang et al. [1] presented results for a fourth scan closer to the antinodal point (cut 2 in Fig.4 of Ref. [1]) than scans (1-3) in Fig. 3 of Ref. [1]. This showed particle-hole symmetry in the accessible energy window near the Fermi energy $(\lesssim 40 \mathrm{meV})$. Note, this energy window is too small to see the actual gap on the particle side. However, the observation that the minimum in the gap is located right at the chemical potential led Yang et al. [1] to suggest that the pseudogap at the antinodal regions has a pairing origin, in agreement with the proposals of Kanigel et al. [4] and Anderson [8]. In a standard pairing theory the energy gap opens up along the Fermi surface whereas in the YRZ propagator the gap opens up at a fixed surface in $\boldsymbol{k}$ space. As such some of use (YRZ) argue that this occurs due to the presence of umklapp particle-particle scattering in addition to the Cooper channel, similar to the case of the half-filled Hubbard 2-leg ladder. [11] As a consequence of this difference, the RVB energy gap, $\Delta^{R}$, acts as a charge gap and only the quasiparticle excitations at the Fermi pockets contribute to the reduced Drude weight and 


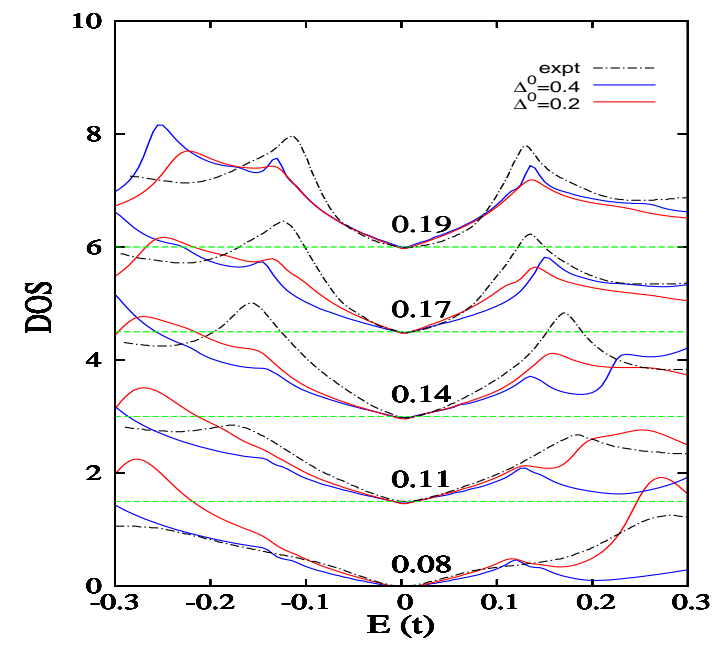

Fig. 4: (Color online) The density of states in the superconducting state for various dopings. The d-wave SC gap is chosen to saturate at ends of Fermi pockets at a fixed value $\left.\Delta_{S}\right|_{\max }=0.14$ to agree with experiments. Two choices for the RVB gap $\Delta_{0}$ are displayed (red $\Delta_{0}=0.2$, blue $\Delta_{0}=0.5$ ). The dashed black curves show the STM data [2] in units of $t_{0}$ $(300 \mathrm{meV})$. The inverse of lifetime $\Gamma=\alpha\left|E_{Q P}\right|$ with $\alpha=0.1$ is used.

reduced coherent stiffness in the superconducting state. That are characteristic of the pseudogap state. [17]

At this point we wish to comment the apparent contradiction between the hole Fermi pockets associated with the YRZ form and the electron Fermi pockets deduced from recent quantum oscillation experiments on underdoped YBCO samples. [18]. These experiments are performed at high magnetic fields and low temperatures whereas the ARPES experiments of Yang et al. [1] were made at zero field and at a relatively high temperature of $140 \mathrm{~K}$. Measurements of the Hall constant on the YBCO samples show a hole-like behavior under the latter conditions but electron-like Hall constant under the conditions of the quantum oscillation experiments [19]. This suggests that the sign change in the Hall constant and the electron pockets, arise from a reconstruction of the Fermi surface [20] at high magnetic fields, possibly related to the similar sign change in the Hall constant that accompanies the superlattice charge and spin order in the static stripe phase. [19]

The recent STM results of Kohsaka et al., [2] requires a generalization of the YRZ propagator to the superconducting state. Originally YRZ added a self-energy term in $G^{R}(\boldsymbol{k}, \omega, x)$. [12] However, during this work we realized that this is appropriate only in the presence of particlehole symmetry. It is more convenient to rewrite Eq1

$$
G^{R}(\boldsymbol{k}, \omega, x)=\sum_{\alpha= \pm} W_{\boldsymbol{k}}^{\alpha} /\left(\omega-E_{\boldsymbol{k}}^{\alpha}\right)+G_{i n c}
$$

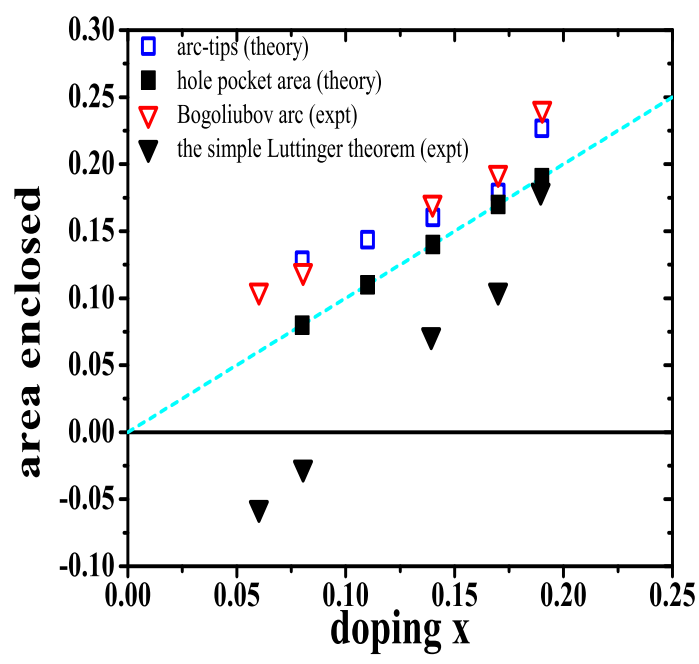

Fig. 5: (Color online): Hole densities implied by areas enclosed by different interpretations of the QP dispersion shown in Fig 3 , and dispersion extrapolated using LDA band structure ( $\mathbf{\nabla}$ from Ref [2]), hole pockets determined by turning points in Fig 3 and lines connecting antinodal points ( $\nabla$ from Ref [2], $\square$ from this work), cyan line and $\mathbf{\square}$ from the YRZ propagator for the Fermi pocket. .

where $E_{\boldsymbol{k}}^{ \pm}=\bar{\xi}_{\boldsymbol{k}} \pm \sqrt{\bar{\xi}_{\boldsymbol{k}}^{2}+\epsilon_{\boldsymbol{k}}^{2}}, \bar{\xi}_{\boldsymbol{k}}=\left(\xi_{\boldsymbol{k}}-\xi_{\boldsymbol{k}}^{(0)}\right) / 2, \epsilon_{\boldsymbol{k}}^{2}=$ $\xi_{\boldsymbol{k}} \xi_{\boldsymbol{k}}^{(0)}+\left|\Delta_{\boldsymbol{k}}^{R}\right|^{2}$ and $\left(W_{\boldsymbol{k}}^{ \pm}\right)^{-1}=1+\left|\Delta_{\boldsymbol{k}}^{R}\right|^{2} /\left(E_{\boldsymbol{k}}^{ \pm}+\xi_{\boldsymbol{k}}^{(0)}\right)^{2}$. The generalization to the superconducting state is straightforward by treating the system as a twoband d-wave superconductor with gap $\Delta_{S}(\boldsymbol{k}, x)=$ $\Delta_{S}(x)\left[\cos \left(k_{x}\right)-\cos \left(k_{y}\right)\right]$ (for simplicity we use a single SC gap) leading to a propagator

$G^{S}(\boldsymbol{k}, \omega, x)=\sum_{\alpha= \pm} W_{\boldsymbol{k}}^{\alpha} /\left[\omega-E_{\boldsymbol{k}}^{\alpha}-\left|\Delta_{S}(\boldsymbol{k}, x)\right|^{2} /\left(\omega+E_{\boldsymbol{k}}^{\alpha}\right)\right]$

with four QPs $E_{i, \boldsymbol{k}}= \pm \sqrt{\left(E_{\boldsymbol{k}}^{\alpha}\right)^{2}+\Delta_{S}(\boldsymbol{k}, x)^{2}}$ with spectral weight $z_{i, \boldsymbol{k}}$.

The superconducting gap opens up along the hole pockets. Kohsaka et al [2] derived the coherent QP dispersion in the superconducting state from the evolution of the interference pattern in the STM spectra measured in a wide surface area as the tunneling voltage is changed in BSCCO samples, ranging from near optimal doping $(x=0.19)$ to strongly underdoped $(x=0.06)$. This determines a set of $\boldsymbol{q}$-vectors connecting the turning points in the isoenergy contours of the QP dispersion. [6,21] These turning points can be compared to the values obtained from Eq15. We use the same values of the parameters $t_{0}, t_{0}^{\prime}$, $t_{0}^{\prime \prime}$ and $J$ as fits to the ARPES spectra at $x=0.12$. Following Zhang et al [14] we assume the RVB gap value $\Delta^{0}(x)\left[=\Delta^{0}\left(1-x / x_{c}\right)\right]$ drops linearly with $x$ with a critical doping $x_{c}=0.20$. Note the value $\Delta^{0}=0.5$ is used in fitting to ARPES results on a sample with $x=0.12$. 
In fitting to the STM results we shall allow for the possibility that the superconducting $\Delta_{S}$ and RVB $\Delta^{0}$ gaps are not independent and that $\Delta^{0}$ may change in the superconducting state from its value in the normal state.

The remaining issue is the form for the superconducting gaps $\Delta_{S}(\boldsymbol{k}, x)$. Generally we expect that $\Delta_{S}(\boldsymbol{k}, x)$ will not continue to grow as $\boldsymbol{k}$ moves beyond the Fermi pockets and the normal state QPs drop below the Fermi energy. Therefore we assume a d-wave form for $\Delta_{S}(\boldsymbol{k}, x)=$ $\Delta^{0}(x)\left(\cos k_{x}-\cos k_{y}\right)$ only for $\boldsymbol{k}$-values on the pockets and set $\Delta_{S}(\boldsymbol{k}, x)$ to be constant beyond the pockets. This results in a two-gap description [22-30] with the antinodal energy gap dominated by the RVB gap at underdoping while the superconducting gap along the hole pockets is more important near optimal doping. We display in Fig 3 the evolution of the contours of constant QP energy near $\mu$. Note the strong variation in QP weight discussed earlier, means that only the outer contours closest to the zone center are relevant. The turning points of these contours are determined by the maxima in the inverse velocity $\left(d E(\boldsymbol{k}) / d k_{\perp}\right)^{-1}$ along these contours. The comparison to the STM results is illustrated in Fig 3 for 4 representative hole densities. We see that the overall agreement is quite satisfactory. Note that the contours defined by the turning points are slightly larger than the underlying hole Fermi pockets and enclose a slightly larger area. This causes the hole density implied by the turning point contour to be slightly larger than the actual hole density. This discrepancy is evident in Fig.3(a) of Kohsaka et al. [2]. The relevant areas are plotted in Fig.5.

Note that in the above analysis we used a standard d-wave form for the superconducting gap, $\Delta_{S}(\boldsymbol{k}, x)=$ $\Delta_{S}^{0}(x)\left(\cos k_{x}-\cos k_{y}\right)$ as observed in ARPES. Kohsaka et al. in their STM experiments found a different angular dependence with a strongly reduced gap near to the nodal directions. The origin of this discrepancy is not clear at present. One possibility could be enhanced forward impurity scattering in the samples used in the STM experiments which Haas et al. showed can lead to a gap suppression near to the nodal directions. [31] However since both techniques are sensitive only to the surface layers it is far from clear that such an explanation is viable.

Kohsaka et al. [2] report an abrupt end to the coherent QP dispersion in the STM experiments when $\boldsymbol{k}$ reaches the umklapp surface. The YRZ propagator contains coherent QP also in the gapped antinodal regions. QP in these regions, however, should be sensitive to the strong local variations in the hole density and the accompanying large variations in the RVB energy gap, $\Delta_{R}^{0}(x)$. [32] This may well lead to localization of the corresponding QP states which lie at energies away from the chemical potential $\mu$. The constant value of $\mu$ makes the Bogoliubov QP states associated with the superconducting gap much less sensitive to disorder. This difference offers a plausible explanation for the change from propagating to localized states that Kohsaka et al. [2] propose takes place as $\boldsymbol{k}$ reaches the umklapp surface.

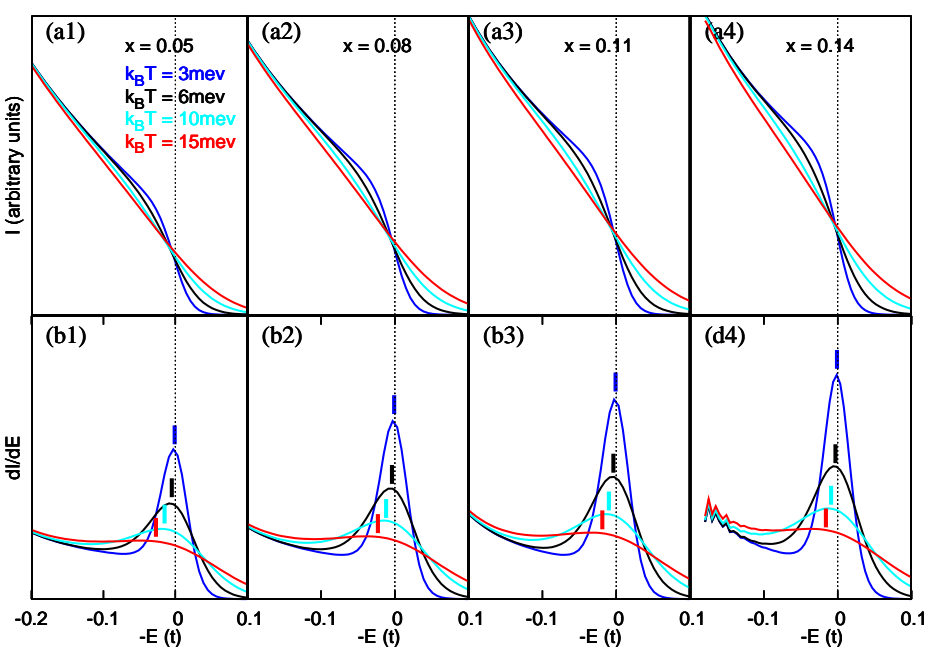

Fig. 6: (Color online): The upper panels are the angle integrated spectra $I(E, T, x)$ near the chemical potential (at zero temperature) for various doping $(0.05-0.14)$. The lower panels are the first order derivative of $I(E, T, x)$, with the peak position $E_{p}$ shifting towards negative electron energy (i.e. positive hole energy), opposite to the shift in the chemical potential. Blue, black, cyan and red curves are for the temperatures $k_{B} T=3,6,10,15 \mathrm{meV}$. The same parameters as shown in Table 1 are used, except $\Delta^{0}=0.4$. Experimental data for $\mathrm{La}_{2} \mathrm{CuO}_{4}$ can be found in Fig.xxx of Hashimoto et al [7].

Kohsaka et al. [2] reported characteristic tunneling spectra, which we can compare to the DOS obtained from the superconducting propagator in $\mathrm{Eq} 5 N(\omega)=$

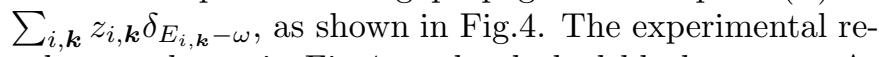
sults are shown in Fig 4 as the dashed black curves. At higher hole densities, $0.1<x<0.19$, the spectra are dominated by the maximum superconducting gap which is larger than the RVB gap, $\Delta^{0}(x)$. As a result the QP bands near the Fermi energy are all split by $\Delta_{S}(\boldsymbol{k})$ with symmetric low energy DOS. At lower hole density the RVB gap $\Delta^{0}(x)$ rises and exceeds the maximum of $\Delta_{S}(\boldsymbol{k})$ leading to two structures in the DOS. One at lower energy related to $\Delta_{S}(\boldsymbol{k}, x)$ at the tips of arc, and second at higher energies associated with the RVB gap. If we keep the RVB gap $\Delta^{0}$ to the value used in the ARPES fits, the DOS shown in blue in Fig 4 displays a much stronger particle-hole asymmetry than the experiments. However, if we reduce the value of $\Delta^{0}$ to 0.2 , the agreement is much improved, suggesting that the two gaps are not independent of each other. Note that the total gap near the antinodal points does not change so much. We do not claim a quantitative fit to the STM DOS but the main features are reproduced at least qualitatively, by the YRZ ansatz.

Next we turn to recent angle integrated photoemission (AIPES) experiments on hole doped $\mathrm{La}_{2} \mathrm{CuO}_{4}$ which showed several anomalous features at underdoping in the normal pseudogap phases. First the total DOS (i.e. angle integrated) show an approximate linear dependence on 


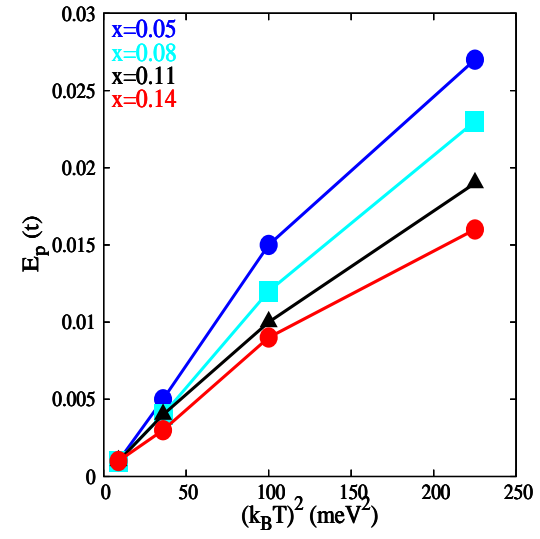

Fig. 7: (Color online): The shifting value of the peak position $E_{p}$ of the derivative of $d I / d E$ towards positive hole energy for varous doping and temperature. It shows a clear quadratic temperature dependence.

the hole energy, $E$, with only a small quadratic correction which grew with increasing $x$. This behavior contrasts with the constant DOS expected from a small hole pocket in a valence band in two dimensions. Secondly, the shift of the peak in the first derivative of the angle integrated spectra $(I(E, T, x)), d I / d E \propto T^{2} / T_{c o h}$ approximately and moves to positive hole energies (not negative). The value of $T_{\operatorname{coh}}(x)$ increases with increasing, $x$. Interestingly, these anomalous features are reproduced by the YRZ model dispersion for quasiparticles. In Fig. 6 (a) we show the results for the angle integrated spectra $I(E, T, x)$ calculated by multiplying the total DOS for quasiparticles by the Fermi function. An approximate linear dependence on $E$ is evident. In Fig. 6 (b) we show that the peak $E_{p}$ of the derivative $|d I / d E|$ moves to positive hole energies opposite to the negative shift of the chemical potential, with a roughly quadratic dependence on the temperature, Fig. 7 The resulting characteristic coherence temperature $T_{c o h}(x)$ increases with increasing $x$. The key anomalous properties of the AIPES spectra are well reproduced by the YRZ form.

The YRZ ansatz for the single particle propagator explains the anomalous properties of the underdoped pseudogap phase as a precursor to the Mott insulating state at stoichiometry. The recent spectroscopic measurements on underdoped BSCCO using ARPES and STM have shed new light on the evolution of the QP properties. In this paper we have shown that the YRZ ansatz provides the basis to understand the key features of the new experimental data such as particle-hole asymmetry, energy dispersion and wave vector dependent spectral weight of normal state QP as well as the coherent QP dispersion and DOS in the superconducting state over a range of hole densities.

We thank Wei-Qiang Chen, Seamus Davis, Carsten Honerkamp, Manfred Sigrist and Alexei Tsvelik for discussions. Support from the MANEP program of the Swiss National funds (K.-Y. Y. and T. M. R.), the US Depart- ment of Energy under Contract No. DE-AC02-98CH10886 (H.-B. Y. and P. D. J.) and RGC grant of HKSAR (K.-Y. $\mathrm{Y}$ and F. C. Z.) is gratefully acknowledged.

\section{REFERENCES}

[1] YAng H.-B. et al., Nature, 456 (2008) 77.

[2] Kohsaka Y. et al, Nature, 454 (2008) 1072.

[3] For a Review see Damascelli A. et al., Rev. Mod. Phys., 75 (2003) 473.

[4] Kanigel A. et al., Nature Physics, 2 (2006) 447.

[5] Norman M. R. et al., Phys. Rev. B, 76 (2007) 174501.

[6] Wang Q. H. And Lee D. H., Phys. Rev. B, 67 (2003) 020511.

[7] Наsнiмото M. et al., arXiv:0806.0101, (unpublished) (2008) .

[8] Anderson P. W. , arXiv:0807.0578, (unpublished) (2008)

[9] Tomeno I. et al., Phys. Rev. B, 49 (1994) 15327.

[10] Konik R. M. et al., Phys. Rev. Lett., 96 (2006) 086407.

[11] Honerkamp C. et al., Phys. Rev. B, 63 (2001) 035109.

[12] Yang K. Y. et al., Phys. Rev. B, 73 (2006) 174501.

[13] Ledermann U. et al.,Phys. Rev. B, 62 (2000) 16383; LeHur K. AND Rice T. M., arXiv:0812.1581, (unpublished) (2008).

[14] Zhang F. C. et al., Supercond. Sci. Techn., 1 (1988) 36.

[15] Anderson P. W. et al., J. Phys. Condens. Matter, 16 (2004) R755-R746.

[16] Ogata M. and Fukuyama H., Rept. Prog. Phys., 71 (2008) 036501.

[17] Basov D. N. And Timusk T., Rev. Mod. Phys., 77 (2005) 721

[18] Doiron-Leyraud N. et al., Nature, 447 (2007) 565

[19] Leboeuf D. et al., Nature, 450 (2007) 533.

[20] Millis A. J. and Norman M. R., Phys. Rev. B, 762007 220503

[21] Bascones E. And Valenzuela B., Phys. Rev. B, $7 \mathbf{7}$ (2008) 024527.

[22] Deutscher G., Nature, 397 (1999) 410.

[23] Tacon M. Le et al., Nature Physics, 2 (2006) 537.

[24] Valenzuela B. and Bascones E., Phys. Rev. Lett., 98 (2007) 227002.

[25] Boyer M. C. et al., Nature Physics, 3 (2007) 802

[26] LEE W. S. et al., Nature, 450 (2007) 81

[27] Hüfner S. et al., Rept. Prog. Phys., 71 (2008) 062501

[28] Illes E. Nicol E. J. And Carbotte J. P., , to be published (2008)

[29] BlanC S. et al., arXiv: 0901.2654, (unpublished) (() 2009)

[30] Kondo T. et al., Nature, 457 (2009) 296

[31] HaAs S. et al., Phys. Rev. B, 56 (1997) 5108.

[32] W.D. Wise et al., Nature Physics, 52009213. 